\title{
Rolling Bearing Incipient Fault Detection Based on a Multi-Resolution Singular Value Decomposition
}

\author{
Jiesi Luo ${ }^{1,2}$ and Shaohui Zhang ${ }^{3, *(1)}$ \\ 1 School of Mechanical and Automotive Engineering, Xiamen University of Technology, Xiamen 361024, \\ China; luojiesi@xmut.edu.cn \\ 2 Key Laboratory of Precision Actuation and Transmission, Fujian Province University, Xiamen 361024, China \\ 3 College of Mechanical Engineering, Dongguan University of Technology, Dongguan 523808, China \\ * Correspondence: zhangsh@dgut.edu.cn
}

Received: 9 September 2019; Accepted: 17 October 2019; Published: 22 October 2019

\begin{abstract}
The periodic impulse characteristics caused by rolling bearing damage are weak in the incipient failure stage. Thus, these characteristics are always drowned out by background noise and other harmonic interference. A novel approach based on multi-resolution singular value decomposition (MRSVD) is proposed in order to extract the periodic impulse characteristics for incipient fault detection. With the MRSVD method, the vibration signal is first decomposed to obtain a group of approximate signals and detailed signals with different resolutions. The first detail signal is mainly composed of noise and the last approximate signal is mainly composed of harmonic interference. Combined with the kurtosis index, the hidden periodic impulse signal will be extracted from the detail signals (in addition to the first detail signal). Thus, the incipient fault detection of a rolling bearing can be fulfilled according to the envelope demodulation spectrum of the extracted periodic impulse signal. The effectiveness of the proposed method has been demonstrated with both simulation and experimental analyses.
\end{abstract}

Keywords: multi-resolution singular value decomposition; rolling bearing; fault detection; demodulation analysis; signal to noise ratio

\section{Introduction}

When a rolling bearing has a local injury in addition to its periodic rotation, the damaged surface will be in contact with other parts that can produce periodic pulse impact, and thus arouse the natural frequency vibration of the inner and outer rings [1,2]. The periodic transient shock signal often occurs in the vibration signal and forms the modulation phenomenon, which is manifested in the frequency spectrum as a modulated side band with equal intervals on both sides of the natural frequency $[1,3]$. Therefore, vibration signals collected from rolling bearings always carry the rich information about machine health conditions [4-6]. Hence, the vibration-based fault detection methods have received intensive study during the past decades.

In order to extract the fault feature information from vibration signals, various signal processing methods have been used in the field of fault detection $[7,8]$, such as empirical mode decomposition (EMD) [9], wavelet transform (WT) [10], and their improved methods [11-14], sparse decomposition [15,16], manifold learning [17], deep learning [18], spectral kurtosis(SK) [19], and envelope analysis [20-24]. These methods realize fault detection from the time domain, frequency domain, or time-frequency domain. In addition to the above methods, there is another kind of method that has been universally used for bearing fault detection to certain success, that is, higher order spectral (HOS) analysis [25-33]. HOS methods such as bispectrum, third order spectrum, etc, were put forward to compensate for the deficiencies of the FFT(Fast Fourier Transform Algorithm)-based techniques when they process the 
non-stationary and non-Gaussian signals. HOS methods realize fault detection from higher-order spectral domains. No matter which domain is selected for bearing fault detection, the objective of the above methods is the detection of the modulation phenomenon formed by the periodic shock characteristics in the resonance band that is excited by the bearing faults, i.e., the resonant demodulation. The reasonable selection of the resonance band will promote the fault detection accuracy of the above methods. However, due to the existence of the background noise and harmonic vibrations in the initial stage of the failure, it is usually difficult to select the optimal frequency band for resonant demodulation. Therefore, the effectiveness of the above resonance demodulation methods is undermined.

Some signal processing methods are applied in order to preprocess the raw vibration signals [12,34-36]. Chibani et al. [37] designed a novel filter in the finite frequency range to overcome the conservatism generated. Chadli et al. [38] proposed a novel fault detection and isolation filter for multi-agent systems. Based on GKYP(generalised Kalman-Yakubovich-Popov) lemma, Zhu et al. [39] proposed a filter to detect faults for vehicle active suspension systems. Li et al. [40] developed a weighted diagnostic observer to optimize the worst case robustness and fault sensitivity simultaneously. The purpose of the preprocessing is to remove the noise and harmonic interference and extract the periodic shock vibration components that are related to the fault for demodulation analysis. In essence, these preprocessing methods decompose the background noise, the harmonic interference, and the periodic shock vibration into different frequency bands. Therefore, the background noise, the harmonic interference, and the periodic shock vibration that are contained in the raw vibration signal should be separable in the frequency domain.

Different types of signals have different singular value characteristics after the singular value decomposition (SVD). Therefore, SVD-based signal processing methods have been proposed, either for noise reduction or classification recognition. The SVD based methods have been also widely used for bearings and rotor-related fault detection to certain success [41-45]. The background noise, the harmonic interference, and the periodic shock vibration have different singular value characteristics after the singular value decomposition (SVD). In contrast to the above frequency-separation-based methods, a new SVD-based preprocessing method, that is, multi-resolution singular value decomposition (MRSVD) [46], is introduced in this study with the objective of extracting the periodic shock vibration from the raw vibration signal which is acquired at the incipient failure stage of a rolling bearing.

Multi-resolution singular value decomposition (MRSVD) is a new signal decomposition method that has been proposed in recent years. Inspired by the multi-resolution analysis idea of wavelet analysis and the construction principle of matrix dichotomy recursion, MRSVD decomposes the analytic signal into a number of approximate and detail signals with different resolutions via the SVD method. As shown in the theoretical analysis in Section 3.1, in the MRSVD decomposition results of a mixed-signal that contains strong background noise, harmonic disturbances, and a periodic shock vibration, the first detail signal is mainly composed of noise, and the last approximate signal is mainly composed of harmonic disturbances. Therefore, the MRSVD method is used to preprocess the rolling bearing vibration signal to remove the background noise signal and the harmonic interference signal. The simulation analysis and the application examples show that the MRSVD method can effectively extract the periodic impact components caused by a bearing fault, even under the influence of strong background noise and harmonic disturbances. Furthermore, the proposed method does not require any prior knowledge about the resonance band. Hence, the MRSVD method has a good application prospect for the initial fault detection of a rolling bearing.

\section{Overview of the Method of Multi-Resolution Singular Value Decomposition}

\subsection{Decomposition Process of MRSVD}

Inspired by the multi-resolution analysis idea of wavelet analysis and the construction principle of matrix dichotomy recursion, a new SVD-based method that is MRSVD is proposed [46]. The specific steps of the algorithm are as follows and the decomposition process is shown in Figure 1. 


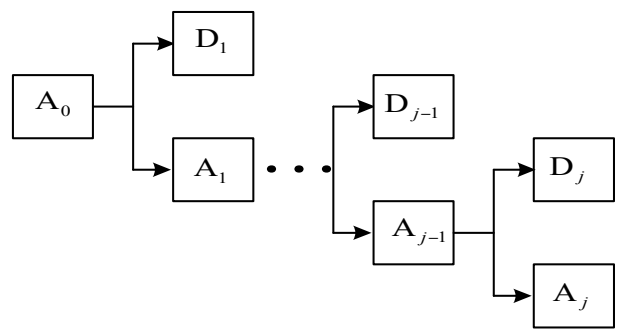

Figure 1. The multi-resolution singular value decomposition (MRSVD) process of a given signal.

Step 1. A Hankel matrix $\mathrm{H}_{0}$ is constructed with two rows for a given signal $\mathrm{A}_{0}=\left(x_{1}, x_{2}, \ldots x_{N}\right)$, i.e., $\mathrm{H}_{0}=\left(\begin{array}{c}x_{1}, x_{2}, \cdots, x_{N-1} \\ x_{2}, x_{3}, \cdots, x_{N}\end{array}\right)$.

Step 2. An SVD is performed on the matrix $\mathrm{H}_{0}$, and on the two obtained singular values that are denoted as $\sigma_{a 1}$ and $\sigma_{d 1}$, with $\sigma_{a 1}>\sigma_{d 1}$. Two signal components can be obtained from $\sigma_{a 1}$ and $\sigma_{d 1}$ via the principle of the SVD matrix's dichotomy recursive algorithm (see the Section 2.2), and these two signal components are denoted as $\mathrm{A}_{1}$ and $\mathrm{D}_{1}$. In terms of the contribution to the original signal, $\mathrm{A}_{1}$ and $\mathrm{D}_{1}$ have different weights. The $\mathrm{A}_{1}$ signal has a larger contribution to the original signal. It is the main component of the original signal and it reflects the main profile obtained from the original signal during the current signal decomposition. Similar to the approximate signal in wavelet analysis, the $\mathrm{A}_{1}$ signal is called as SVD approximate signal. In addition, the $\mathrm{D}_{1}$ signal reflects the details obtained from the original signal during the current signal decomposition. The $\mathrm{D}_{1}$ signal is similar to the detail signal in the wavelet analysis, so it is called the SVD detail signal.

Step 3. The detail signal $D_{1}$ is preserved, and the construction of the 2-row Hankel matrix $\mathrm{H}_{1}$ is continued for the $\mathrm{A}_{1}$ approximate signal in order to obtain the next level singular value decomposition. Consequently, a series of SVD detail signals and approximate signals can be obtained using the recursion decomposition layer by layer.

\subsection{Principle of the SVD Matrix's Dichotomy Recursive Algorithm}

Presuming that the $(j-1)$ th MRSVD decomposition of the given signal $\mathrm{A}_{0}=\left(x_{1}, x_{2}, \ldots x_{N}\right)$ has been carried out, the $(j-1)$ th SVD approximate signal will have also been obtained and denoted as $\mathrm{A}_{j-1}=\left(a_{j-1,1}, a_{j-1,2}, \ldots \mathrm{n} a_{j-1, N}\right)$. A 2-row Hankel matrix $\mathrm{H}_{j}$ can be constructed, i.e., $\mathrm{H}_{j}=\left(\begin{array}{c}a_{j-1,1}, a_{j-1,2}, \cdots, a_{j-1, N-1} \\ x_{j-1,2}, x_{j-1,3}, \cdots, x_{j-1, N}\end{array}\right)$.

The SVD result of the matrix $\mathrm{H}_{j}$ can be expressed as

$$
\mathrm{H}_{j}=\mathrm{U}_{j} S_{j} \mathrm{~V}_{j}
$$

where $\mathrm{U}_{j}=\left(u_{j 1}, u_{j 2}\right), \mathrm{U}_{j} \in \mathrm{R}^{2 \times 2}, \mathrm{~V}_{\mathrm{j}}=\left(v_{j 1}, v_{j 2}, \ldots, v_{j(N-1)}\right), \mathrm{V}_{\mathrm{j}} \in \mathrm{R}^{(N-1) \times(N-1)}$, and $\mathrm{U}_{j}$ and $\mathrm{V}_{j}$ are the left and right orthogonal matrices obtained by the $j$ th decomposition. $S_{j}=\left(\operatorname{diag}\left(\sigma_{a j}, \sigma_{d j}\right), 0\right), S_{j} \in R^{2 \times(N-1)}$, and $\sigma_{a j}$ and $\sigma_{\mathrm{d} j}$ are the approximate singular value and the detail singular value obtained using the $j$ th decomposition.

In order to obtain the SVD approximate signal $\mathrm{A}_{j}$ and the detail signal $\mathrm{D}_{j}$ for the $j$ th decomposition, $\mathrm{H}_{j}$ in the formula (1) can be expressed as the following form.

$$
\mathrm{H}_{j}=\sigma_{a j} u_{j 1} v_{j 1}^{T}+\sigma_{d j} u_{j 2} v_{j 2}^{T}
$$

where $u_{j 1} \in \mathrm{R}^{2 \times 1}, v_{j 1} \in \mathrm{R}^{(N-1) \times 1}$, and $i=1$,2. Denoting $\mathrm{H}_{a j}=\sigma_{a j} u_{j 1} v_{j 1}^{T}$, then $\mathrm{H}_{a j} \in \mathrm{R}^{2 \times(N-1)}$ and $\mathrm{H}_{a j}$ corresponds to the larger singular value $\sigma_{a j}$, which reflects the body of the original signal, so it is called the approximation matrix. In addition, $\mathrm{H}_{\mathrm{d} j}=\sigma_{d j} u_{j 2} v_{j 2}^{T}, \mathrm{H}_{d j} \in \mathrm{R}^{2 \times(N-1)}$, and $\mathrm{H}_{d j}$ corresponds to the smaller singular value $\sigma_{d}$, which reflects the detail of the original signal, so it is called the detail matrix. 
The SVD approximate signal $\mathrm{A}_{j}$ and the detail signal $\mathrm{D}_{j}$ are obtained from $\mathrm{H}_{a j}$ and $\mathrm{H}_{d j}$, respectively, and the specific process is described as follows.

Both of the matrixes, $\mathrm{H}_{a j}$ and $\mathrm{H}_{d j}$, can be expressed in the form of row vectors, i.e., $\mathrm{H}_{a j}=\left(\begin{array}{c}\sigma_{a j} u_{j 1,1} v_{j 1}^{T} \\ \sigma_{a j} u_{j 1,2} v_{j 1}^{T}\end{array}\right)$ and $\mathrm{H}_{d j}=\left(\begin{array}{c}\sigma_{d j} u_{j 2,1} v_{j 2}^{T} \\ \sigma_{d j} u_{j 2,2} v_{j 2}^{T}\end{array}\right)$. The specific row vector forms of the $\mathrm{H}_{a j}$ and $\mathrm{H}_{d j}$ matrixes are displayed in Figure $2 \mathrm{a}, \mathrm{b}$.

(a)

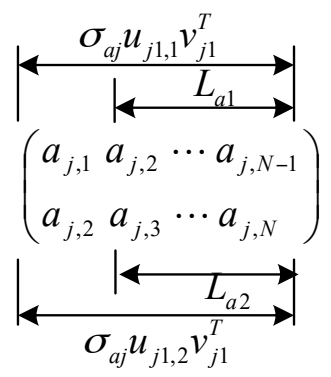

(b)

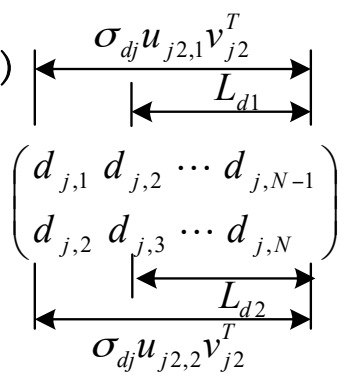

Figure 2. The specific row vector forms of the $\mathrm{H}_{a j}$ and $\mathrm{H}_{d j}$ matrixes. (a) The specific row vector forms of the $\mathrm{H}_{a j}$ matrixes. (b) The specific row vector forms of the $\mathrm{H}_{d j}$ matrixes.

Observably, both $\mathrm{L}_{a, 1}$ and $\mathrm{L}_{a, 2}$, as shown in Figure $2 \mathrm{a}$, represent the data points $a_{j, 1}, a_{j, 2}, \ldots, a_{j, N-1}$ of the SVD approximate signal $\mathrm{A}_{j}$. However, $\mathrm{L}_{a, 1}$ is not equal to $\mathrm{L}_{a, 2}$. In this study, all of the elements in matrix $\mathrm{H}_{a j}$ that represent the same data in signal $\mathrm{A}_{j}$, are averaged and the acquired mean value of the corresponding data of $\mathrm{A}_{j}$ is used. Therefore, the SVD approximate signal $\mathrm{A}_{j}$ is obtained using Formula (3).

$$
\mathrm{A}_{j}=\left(a_{j, 1},\left(\mathrm{~L}_{a, 1}+\mathrm{L}_{a, 2}\right) / 2, a_{j, N}\right)
$$

In a similar manner to the above description, the SVD detail signal $\mathrm{D}_{j}$ is obtained using Formula (4).

$$
\mathrm{D}_{j}=\left(d_{j, 1},\left(\mathrm{~L}_{d, 1}+\mathrm{L}_{d, 2}\right) / 2, d_{j, N}\right)
$$

\section{Application of the MRSVD Method in the Incipient Fault Detection of the Rolling Bearings}

\subsection{Study of the Principle of the Noise Cancellation and Anti-Harmonic Interference of the MRSVD Method}

The rolling bearing vibration signal usually contains three signal components, including the periodic shock component caused by the fault, the harmonic component generated by the vibration of the equipment or the harmonic current, and the background noise component. Supposing that the gathered vibration signal of the rolling bearing is $x(i)$, then $x(i)$ can be expressed as Formula (5).

$$
x(i)=s(i)+n(i)+h(i) \quad i=1,2, \cdots, N
$$

In Formula (5), $s(i)$ is the periodic shock signal, $n(i)$ is the background noise signal, $h(i)$ is the harmonic signal, and $N$ is the length of the signal.

According to Formula (5), The Hankerl matrix $\mathrm{H}$ constructed by the signal $x(i)$ can be expressed as Formula (6).

$$
\mathrm{H}=\mathrm{H}_{\mathrm{s}}+\mathrm{H}_{n}+\mathrm{H}_{h}
$$

where, $\mathrm{H}_{s}, \mathrm{H}_{n}$, and $\mathrm{H}_{h}$ are the Hankerl matrixes constructed by the signals $s(i), n(i)$, and $h(i)$, respectively. For the MRSVD method, the number of rows in the Hankerl matrix is 2. In this type of matrix, the current row vector lags only one data point behind the previous row vector. The SVD of the 2-row Hankerl matrix for these three types of signals was analyzed as follows:

(1) For the harmonic signal $h(i)$, the 2-row Hankerl matrix $\mathrm{H}_{h}$ was highly correlated, so the rank of the matrix was 1 . It was assumed that the two singular values obtained using the SVD of $\mathrm{H}_{h}$ were $\sigma_{s}$ and $\xi$, i.e., $\sigma\left(\mathrm{H}_{s}\right)=\left(\sigma_{s}, \xi\right)$, so $\xi$ was a small positive number and $\sigma_{s} \gg \xi$. The energy of the harmonic 
signal $h(i)$ was mainly distributed to the first singular value, and only a tiny fraction was distributed to the second singular value.

(2) For the noise signal $n(i)$, its auto-correlation function was $R_{n}(\tau)=d^{2} \delta(\tau), d$ was the standard deviation of the signal $n(i)$, and $\delta(\tau)$ was the unit impulse function. For the 2-row Hankerl matrix $\mathrm{H}_{n}$, although its two adjacent row vectors were only one bit behind each other, they were uncorrelated, so the rank of the matrix was 2. Therefore, the correlation of the 2-row Hankerl matrix $\mathrm{H}_{n}$ was nearly equal, which was denoted as $\sigma_{n}$, i.e., $\sigma\left(\mathrm{H}_{n}\right)=\left(\sigma_{n}, \sigma_{n}\right)$.

(3) For the periodic shock signal $s(i)$, the 2-row Hankerl matrix $\mathrm{H}_{s}$ had a certain correlation. However, compared to the correlation of the 2-row Hankerl matrix $\mathrm{H}_{h}$, the correlation of the 2-row Hankerl matrix $\mathrm{H}_{s}$ was lower. The correlations of the 2-row Hankerl matrix $\mathrm{H}_{s}$ were denoted as $\sigma_{h b}$ and $\sigma_{h s}$, i.e., $\sigma\left(\mathrm{H}_{h}\right)=\left(\sigma_{h b}, \sigma_{h s}\right)$, and $\sigma_{h b}>\sigma_{h s}$.

Based on the above analysis, the singular value matrix $\mathrm{H}$ constructed by the signal $x(i)$ satisfied Formula (7).

$$
\begin{aligned}
\sigma(H) & \leq \sigma\left(H_{s}\right)+\sigma\left(H_{n}\right)+\sigma\left(H_{\mathrm{h}}\right) \\
= & \left(\sigma_{s}+\sigma_{n}+\sigma_{h b}, \xi+\sigma_{n}+\sigma_{h s}\right)
\end{aligned}
$$

In fact, since the number of rows of the singular value matrix $\mathrm{H}$ was only 2 , thus $\sigma(\mathrm{H}) \approx\left(\sigma_{s}+\sigma_{n}+\sigma_{h b}\right.$, $\left.\xi+\sigma_{n}+\sigma_{h s}\right)$. The energies of the approximate signal and the detail signal were proportional to the square of the singular value. From Formula (7), it can be seen that the singular value corresponding to the detailed signal $D_{1}$ can be represented as $\xi+\sigma_{n}+\sigma_{h s}$. The main components of the detailed signal $\mathrm{D}_{1}$ were the noise signal and a small amount of the periodic shock signals. The total energy of the noise signal contained in the detailed signal $\mathrm{D}_{1}$ accounted for half of the total noise energy contained in the signal $x(i)$. From Formula (7), it also can be seen that the singular value corresponding to the approximate signal $\mathrm{A}_{1}$ can be represented as $\sigma_{s}+\sigma_{n}+\sigma_{h b}$. Similar to the signal $x(i)$, the approximate signal $\mathrm{A}_{1}$ also contained noise components, harmonic components, and periodic shock components. Continuing to the next level of decomposition of the MRSVD, the detailed signal $\mathrm{D}_{2}$ was obtained. Similar to signal $\mathrm{D}_{1}$, the signal $\mathrm{D}_{2}$ also contained the noise signal and a small part of the periodic impact signal. The total energy of the noise signal contained in $\mathrm{D}_{2}$ accounted for half of the total energy of the noise contained in $\mathrm{A}_{1}$. With the increase of the number of decomposition layers, the energy of the noise was halved. The total energy of the noise contained in $\mathrm{D}_{j}$ was half the amount of energy contained in $\mathrm{D}_{j-1}$. As the noise decreased, the periodic shock components were highlighted. Nevertheless, for the harmonic signal $h(i)$, only a very small amount of energy $\left(\xi^{2} /\left(\sigma_{s+} \xi\right)^{2} \rightarrow 0\right)$ was decomposed into the detail signal in each decomposition level of the MRSVD. Therefore, most of the energy of the harmonic signal $h(i)$ was retained in the last layer of the approximation signal.

Based on the above analysis, for a rolling bearing signal that is affected by strong background noise and harmonic interference, the first detailed signal $\mathrm{D}_{1}$ obtained by its decomposition will be mainly a noise signal, and its kurtosis value will be near 3 . With the increase of the number of decomposition layers, the noise components will reduce layer by layer, and the periodic impact composition will increase layer by layer. Therefore, the kurtosis value will increase with the increase of the number of decomposition layers. When the kurtosis value reaches a certain maximum, it will decrease with the increase of the number of decomposition layers. This is because the periodic impact components contained in the residual approximation signal also decrease layer by layer, which will be confirmed in the simulation analysis.

\subsection{Determination of the Number of Decomposition Layers of MRSVD}

According to the analysis in Section 3.1, if the number of decomposition layers of the MRSVD is too small, the detailed signal obtained will contain more noise interference. If there are a large number of decomposition layers, the multiple decomposition layers will lead a larger calculation, which is inconsistent with the real-time requirement of the fault detection of a rolling bearing. However, if the number of decomposition layers is too large, the decomposed results will generate spurious signals. Therefore, the number of decomposition layers of the MRSVD in this study is adaptively obtained in 
the decomposition process when the kurtosis value of the detail signal reached a maximum. Therefore, if the current approximate signal continued to decompose, the kurtosis value of the next layer of detail signals would show a downward trend, so the number of decomposed layers was determined to be the current number of decomposed layers plus five.

\section{Simulation Studies}

In order to verify the effectiveness of the MRSVD in the aspects of rolling bearing incipient fault detection, the mathematical model shown in Formula (8) was used to simulate the early fault vibration signal of a rolling bearing

$$
\begin{aligned}
x(t) & =s(t)+n(t)+h(t) \\
& =\sum_{m=-M}^{M} A_{m} e^{-\beta\left(t-m T_{p}-\sum_{i=-M}^{m} \tau_{i}\right)} \sin \left(\omega_{r}\left(t-m T_{p}-\sum_{i=-M}^{M} \tau_{i}\right)\right) u\left(t-m T_{p}-\sum_{i=-M}^{m} \tau_{i}\right) \\
& +n(t)+h(t)
\end{aligned}
$$

where $n(t)$ is the noise signal added by the MATLAB function awgn.m, the signal-to-noise ratio (SNR) is set to -6 , and $h(t)$ is the harmonic interference signal that contains $N$ harmonic signals, which can be expressed as $h(t)=\sum_{j=1}^{N} B_{j} \sin \left(2 \pi f_{i} t\right)$, i.e., using the amplitude and the frequency of the $j$ th harmonic signal, which are $B_{j}$ and $f_{j}$, respectively. The periodic shock signal caused by the fault contains $2 \mathrm{M}+1$ impulses. In Formula (8), $A_{m}$ is the amplitude of the $m$ th shock, $\beta$ is the decay coefficient caused by the structural damping, $T_{p}$ is the time period corresponding to the fault characteristic frequency, i.e., the fault feature frequency, $f c=1 / T_{p} . u(t)$ is the unit impulse function, $\omega_{r}$ is the excited frequency, and $\tau_{i}$ is the random variable whose mean value is zero and whose variance is between $\left(0.01 T_{p}, 0.02 T_{p}\right)$, which is the effect of the random slippage of the rollers.

The parameters of the simulated signal are displayed in Table 1 . The noise was directly added to the simulated periodic impulse signal before the harmonic interference signals were added. Therefore, the signal-to-noise ratio (SNR) in Table 1 is relative to the simulated periodic signal. The simulation signal was sampled at 20,000 samples $s^{-1}$, and the number of sampling points was set to 20,000. After sampling, the simulated signal, along with the noise, the harmonic interference signals, and their Hilbert demodulation spectrums, are plotted in Figure 3. Due to the influences of the noise and the harmonic interference, it is impossible to judge the fault of rolling bearing from Figure 3a. In Figure 3b, the frequency differences of the harmonic components contained in the harmonic signal are mistakenly identified as the demodulation frequencies. However, the actual fault characteristic frequencies are not highlighted in Figure 3b.

Table 1. Parameters of the simulated signal. Signal-to-noise ratio (SNR).

\begin{tabular}{cccccccccccc}
\hline$A_{m}$ & $\boldsymbol{T}_{\boldsymbol{P}}$ & $\boldsymbol{M}$ & $\boldsymbol{\beta}$ & $\boldsymbol{\omega}_{\boldsymbol{r}}$ & $\mathrm{SNR}$ & $\boldsymbol{B}_{\mathbf{1}}$ & $\boldsymbol{f}_{\mathbf{1}}$ & $\boldsymbol{B}_{\mathbf{2}}$ & $\boldsymbol{f}_{\mathbf{2}}$ & $\boldsymbol{B}_{\mathbf{3}}$ & $\boldsymbol{F}_{\mathbf{3}}$ \\
\hline \multirow{2}{*}{1} & \multirow{2}{*}{$0.008 \mathrm{~s}$} & \multirow{2}{*}{250} & $\begin{array}{c}1500 \\
\mathrm{~Hz}\end{array}$ & $\begin{array}{c}2048 \\
\mathrm{~Hz}\end{array}$ & -6 & \multirow{2}{*}{1.35} & $60 \mathrm{~Hz}$ & \multirow{2}{*}{1.4} & 230 & \multirow{2}{*}{1.5} & 800 \\
& & & & & & & & & & &
\end{tabular}

(a)

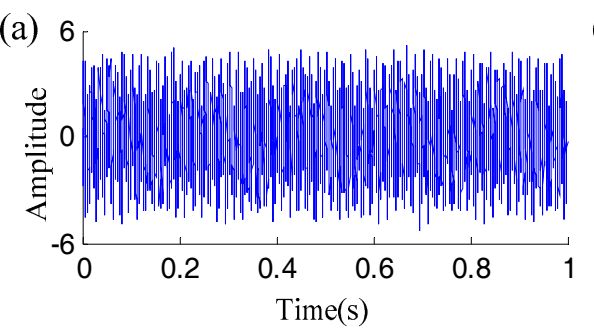

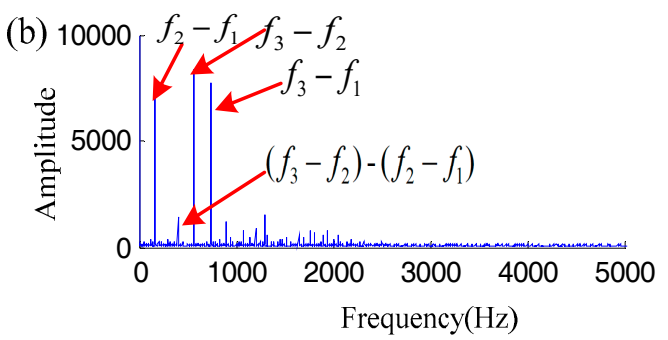

Figure 3. Simulated signal (with noise and interference) and its Hilbert demodulation spectrum. (a) The waveform of the simulted signal in the time-domain. (b) The Hilbert demodulation spectrum of the simulted signal. 
The 2-row Hankerl matrixes were constructed for the simulation signal and their periodic impact, noise, and resonance components are denoted as $\mathrm{H}, \mathrm{H}_{\mathrm{s}}, \mathrm{H}_{\mathrm{n}}$, and $\mathrm{H}_{\mathrm{h}}$, respectively. The result singular values of the SVD of the matrix $\mathrm{H}, \mathrm{H}_{\mathrm{s}}, \mathrm{H}_{\mathrm{n}}$, and $\mathrm{H}_{\mathrm{h}}$ are displayed in Table 2. The numerical results shown in Table 2 are consistent with the singular value analysis of the three kinds of signals (the periodic shock signal, the noise signal, and the harmonic signal) in Section 3.1 of this manuscript. To be specific, the two singular values obtained by the decomposition of the harmonic signals are quite different from each other, the two singular values obtained by the decomposition of noise signals are nearly equal, and the singular values obtained by the decomposition of periodic shock waves are different. However, the difference between them is neither too large nor close to zero.

Table 2. Result singular values of the singular value decomposition SVD of matrix $H, H_{s}, H_{n}$, and $H_{h}$.

\begin{tabular}{|c|c|c|c|c|c|}
\hline$\sigma$ & Hankerl Matrix & $\mathbf{H}$ & $\mathbf{H}_{\mathrm{s}}$ & $H_{n}$ & $\mathbf{H}_{\mathrm{h}}$ \\
\hline & $\sigma_{a}$ & 354.8617 & 44.5982 & 69.4838 & 346.2494 \\
\hline & $\sigma_{d}$ & 73.3264 & 14.2705 & 66.8183 & 17.5887 \\
\hline
\end{tabular}

The simulated signal shown in Figure 3 was decomposed using the MRSVD method, and the number of decomposed layers was set to 15 . The singular values and the kurtosis coefficients corresponding to the obtained detail signals of each layer are shown in Figure $4 a, b$.

(a)

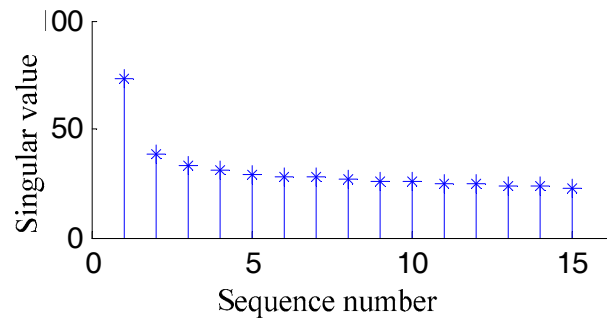

(b) 3.5

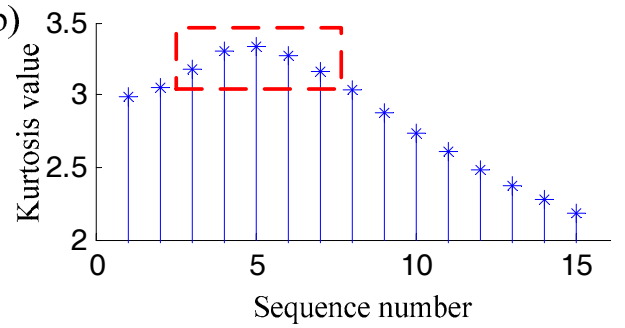

Figure 4. The singular values and kurtosis coefficients corresponding to the obtained detail signals of each layer. (a) The singular values corresponding to the obtained detail signals of each layer. (b) The kurtosis coefficients corresponding to the obtained detail signals of each layer.

It can be seen from Figure 4a that the singular values of the first several detail signals decreased rapidly because the noise was removed relatively quickly at the beginning. Then the singular values of the detail signals decreased gradually with the increase of the number of layers. To be specific, the noise removal became slow with the increase of the number of decomposed layers.

As shown in Figure $4 b$, because the first detail signal contained a large amount of noise, its kurtosis coefficient was nearly equal to 3 . As the number of decomposed layers increased, the kurtosis value also increased, because the noise component contained in the detail signal decreased layer by layer and the periodic impact component increased layer by layer. When the number of decomposed layers was 5 , the kurtosis value of the detail signal reached a maximum, and the kurtosis value decreased with the increase of the decomposed layer because the periodic shock component contained in the residual approximate signal decreased layer by layer. Therefore, the conclusions pertaining to the kurtosis value analysis of the detail signals in Section 3.1 are verified in Figure $4 \mathrm{~b}$.

Figure 5 shows the time domain and frequency domain waveforms of the first detail signal obtained by the decomposition of the simulated signal via the MRSVD method. The main component of the signal in Figure 5 is the noise signal. Therefore, there is no highlighted frequency in the frequency spectrum of Figure 5. The time-domain and frequency-domain waveforms of the last layer of the approximate signal obtained by the decomposition of the simulated signal via the MRSVD method are plotted in Figure 6a,b. As stated in Section 3.1, most of the energy of the harmonic signal was 
retained in the last layer of the approximation signal, so the highlighted frequencies in Figure $6 \mathrm{~b}$ are the harmonic frequencies $f_{1}, f_{2}$ and $f_{3}$.

(a)

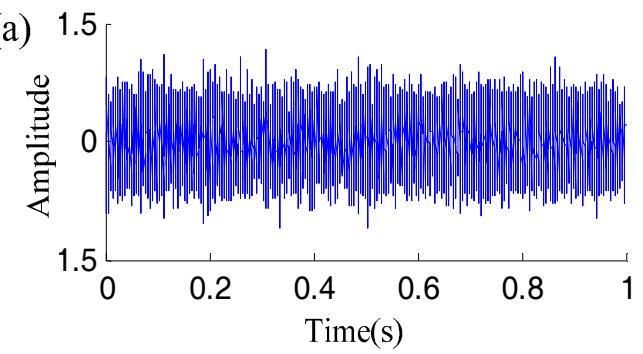

(b) 400

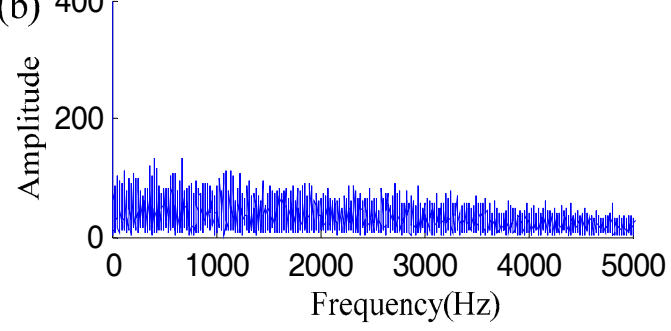

Figure 5. The time and frequency waveform of the first detail signal. (a) The waveform of the first detail signal in the time-domain. (b) The waveform of the first detail signal in the frequency-domain.
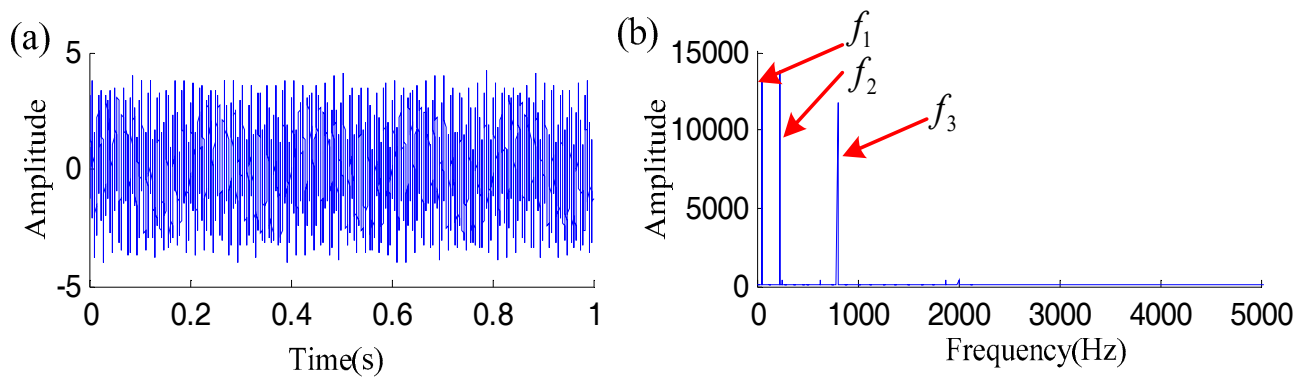

Figure 6. The time and frequency waveform of the last layer approximation signal. (a) The waveform of the last layer approximation signal in the time-domain. (b) The waveform of the last layer approximation signal in the frequency-domain.

The five signals with maximum kurtosis were selected (see the red box in Figure $4 \mathrm{~b}$ ), and these five signals were added together in order to form a synthetic signal. Figure 7 shows the time-domain waveform of the synthetic signal and its Hilbert envelope demodulation spectrum. Although some noise was also contained in the synthetic signal, the fault characteristic frequency and its multiples are highlighted in the Hilbert envelope demodulation spectrum shown in Figure $7 \mathrm{~b}$.
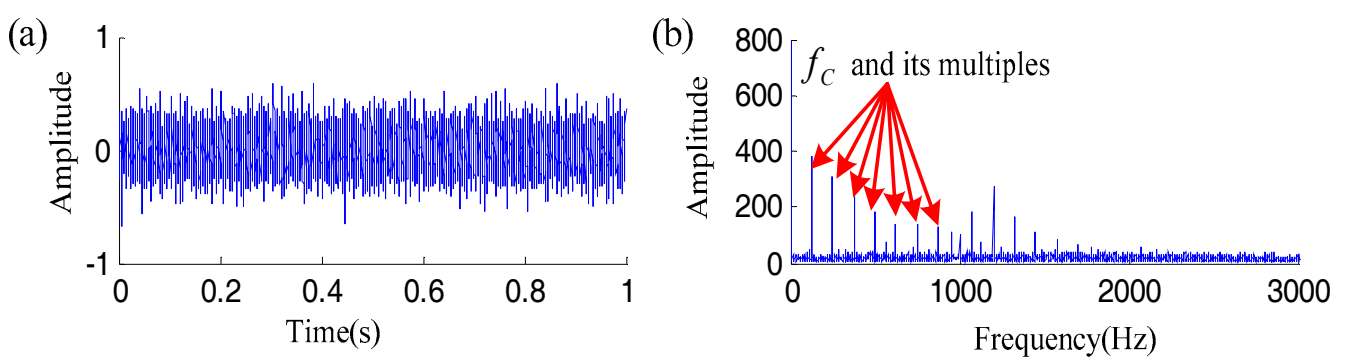

Figure 7. Time and Hilbert envelope demodulation spectrum of the extracted periodic impulse signal. (a) The waveform of the extracted periodic impulse signal in the time-domain. (b) The Hilbert envelope demodulation spectrum of the extracted periodic impulse signal.

In the above simulation analysis, the number of decomposed layers of the MRSVD method was set to 15 (Figure $4 \mathrm{~b}$ ). The corresponding sequence number of the detail signal with the maximum kurtosis value is 5 . Therefore, when combined with the description of the decomposition layer number settings in Section 3.2, the number of decomposed layers of the MRSVD method only needed to be set to 10 . 


\section{Experiment Studies}

In order to verify the effectiveness of the MRSVD method in practical application, vibration signals of the outer race fault rolling bearing were collected from the experimental setup shown in Figure 8 . The signal from the sensor (ICP ${ }^{\mathrm{R}}$ accelerometer, model 623C01) is fed to an NI data acquisition module (NI USB-6212 BNC) and is recorded by the computer with the LABVIEW software. The sampling frequency is $20 \mathrm{kHz}$ and acquired data length is $40 \mathrm{k}$. The fault characteristics of incipient failure are not obvious; the periodic shock vibration associated with faults is often drowned in the background noise and the harmonic interference. Therefore, a more challenging condition is created at the experiment; the sensor signal conditioner is removed. Hence, the raw signal from sensor is not altered and not amplified. Obviously, the signal collected in this way is less clean and is considered more realistic. In the raw data, the interference, such as the power line frequency around $60 \mathrm{~Hz}$, cannot be ignored. The background noise will be more obvious. For example, the noise caused by casualization error of the NI data acquisition module is inevitable and becomes relatively stronger when the raw signal voltage of the accelerometer output is very low.

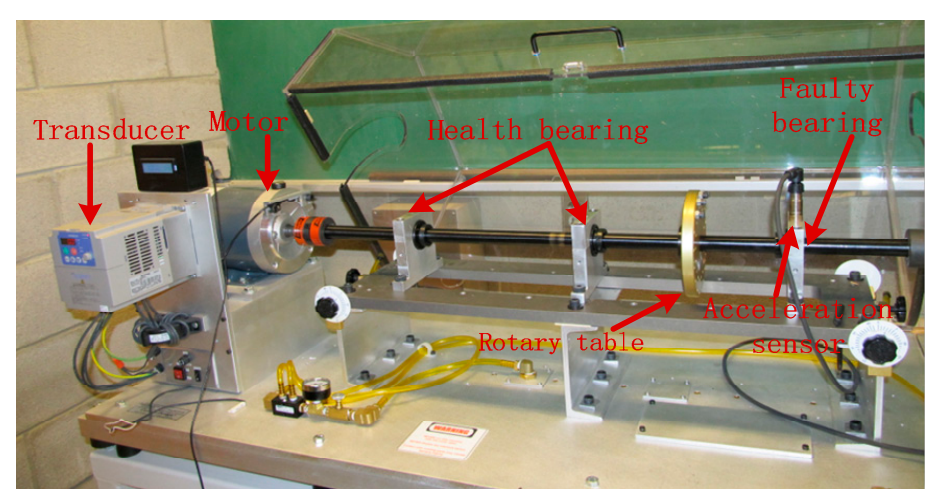

Figure 8. The test rig.

The ER16K ball bearings, manufactured by Rexnord Industries, are used throughout the experiments. The bearing parameters are shown in Table 3. The outer race fault is in the top position, see Figure 9. In Table 3, the Ball Pass Frequency Outer Race (BPFO) and $f_{\mathrm{r}}$ terms represent the characteristic frequency of outer race failure and the rotation frequency of the rotating shaft, respectively. To be specific, the frequency of the rotary shaft rotation frequency $f_{\mathrm{r}}$ is the resonance harmonic signal caused by the imbalance of the rotary table.

Table 3. The related parameters of the outer fault bearing. Ball Pass Frequency Outer Race (BPFO).

\begin{tabular}{ccccc}
\hline Type & Roll diameter & Pitch diameter & Number of rolls & BPFO \\
\hline ER16K & $7.94 \mathrm{~mm}$ & $38.52 \mathrm{~mm}$ & 9 & $3.572 f_{r}$ \\
\hline
\end{tabular}

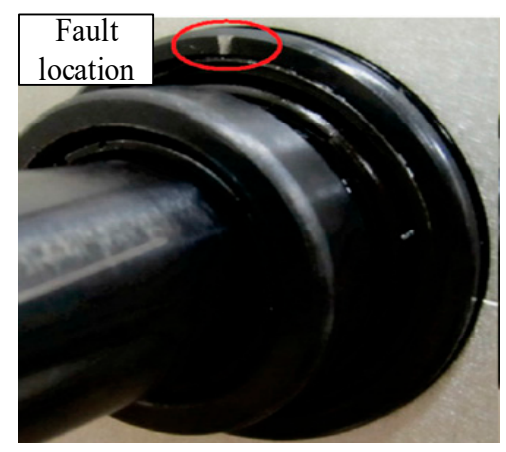

Figure 9. The fault bearing used in the experiment. 
The ER16K ball bearings, manufactured by Rexnord Industries, are used throughout the experiments. The bearing parameters are shown in Table 3. The outer race fault is in the top position, see Figure 9. In Table 3 , the BPFO and $f_{\mathrm{r}}$ terms represent the characteristic frequency of outer race failure and the rotation frequency of the rotating shaft, respectively. To be specific, the frequency of the rotary shaft rotation frequency $f_{\mathrm{r}}$ is the resonance harmonic signal caused by the imbalance of the rotary table.

The rotation speed of the rotation axis was $1440 \mathrm{rpm}$, so $f_{\mathrm{r}}=12 \mathrm{~Hz}$ and $\mathrm{BPFO}=85.728 \mathrm{~Hz}$. The time domain waveform and the Hilbert envelope demodulation spectrum of the collected vibration signal with the noise and the resonance interference are shown in Figure 10a,b. For Figure 10b, the display interval of the coordinate axis is reduced, and a detailed graph is obtained in order to see the details shown in Figure 11. The resonant frequency of $24 \mathrm{~Hz}$ and the power line frequency of $60 \mathrm{~Hz}$ can be clearly seen in Figure 11. However, the failure characteristic frequency of the bearing outer race cannot be seen.
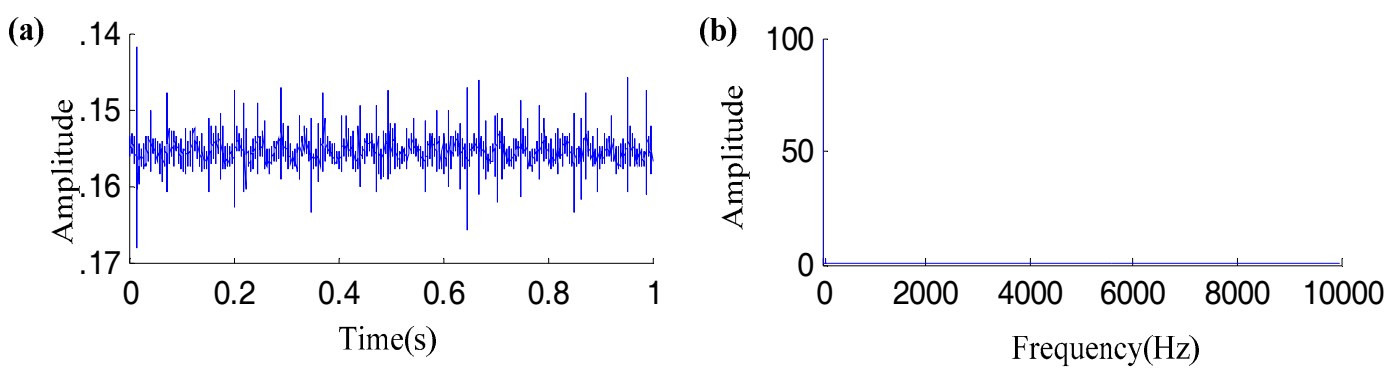

Figure 10. Time domain waveform and Hilbert envelope demodulation spectrum of the collected vibration signal with noise and resonance interference. (a) The waveform of the collected vibration signal in the time-domain. (b) The Hilbert envelope demodulation spectrum of the collected vibration signal.

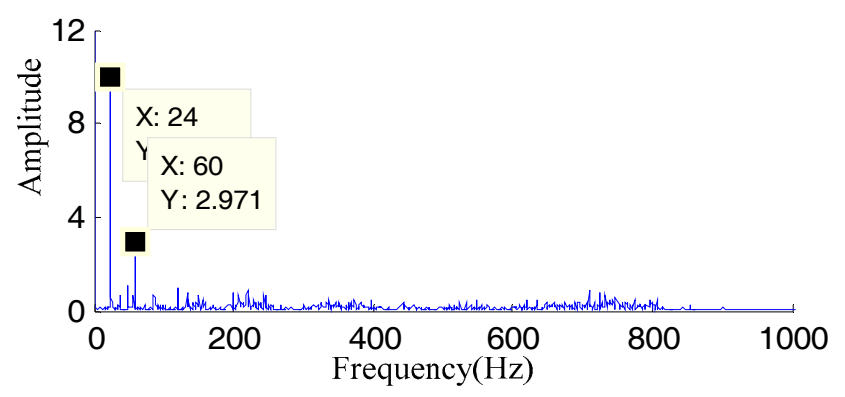

Figure 11. Details of Figure 9b from zooming out of the display range.

The measured signal shown in Figure 10a is decomposed by MRSVD. The time domain waveform and the Hilbert envelope demodulation spectrum of the detail signal with the maximum kurtosis value are displayed in Figure 12a,b. The periodic shock oscillations with single-sided attenuations which were caused by the bearing failure can be seen in Figure 12a. The characteristic frequency of the outer race failure (BPFO) and its double are highlighted in Figure 12b.

The five detail signals with maximum kurtosis values obtained using MRSVD decomposition are plotted in Figure 13d1-d5. Furthermore, their corresponding Hilbert envelope demodulation spectrums are shown in Figure 13h1-h5. In Figure 13h1-h5, in order to display the fault feature frequency more clearly, the display scope in the frequency domain is limited to the range of $0 \mathrm{~Hz}$ to $800 \mathrm{~Hz}$. The time domain waveform and the Hilbert envelope demodulation spectrum of the detail signal with the maximum kurtosis value are also displayed in Figure 13d1,h1 in order to provide a comparison with the rest of the figures in Figure 13. Similarly to Figure 11, the periodic shock oscillations with single-sided attenuations caused by the bearing failure can also be seen in Figure 13d1-d5. The characteristic frequency of the outer race failure (BPFO) and its double are also highlighted in Figure 13h2-h5. Therefore, it can be determined that outer race fault exists in the roll bearing. 

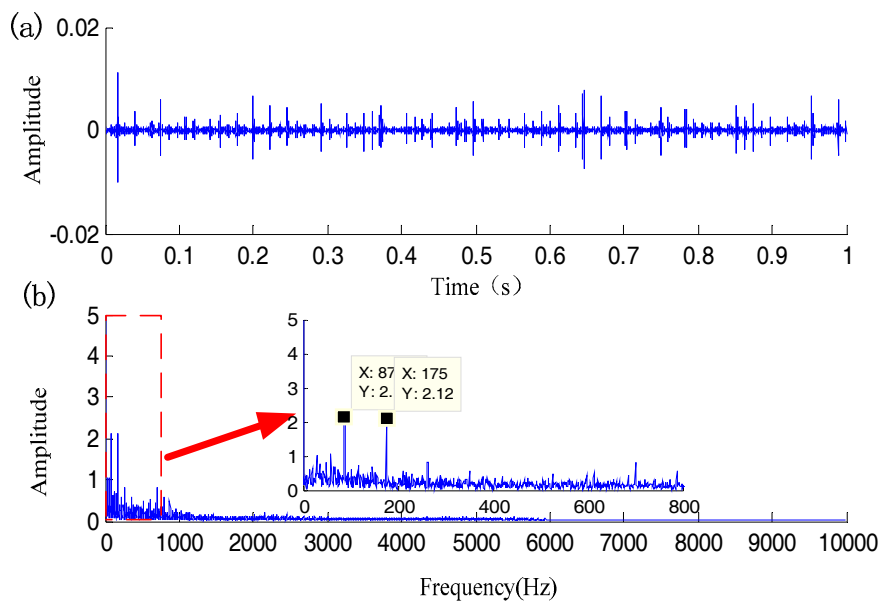

Figure 12. Time domain waveform and Hilbert envelope demodulation spectrum of the detail signal with the maximum kurtosis value. (a) The waveform of the detail signal with the maximum kurtosis value. (b) The Hilbert envelope demodulation spectrum of the detail signal with the maximum kurtosis value.
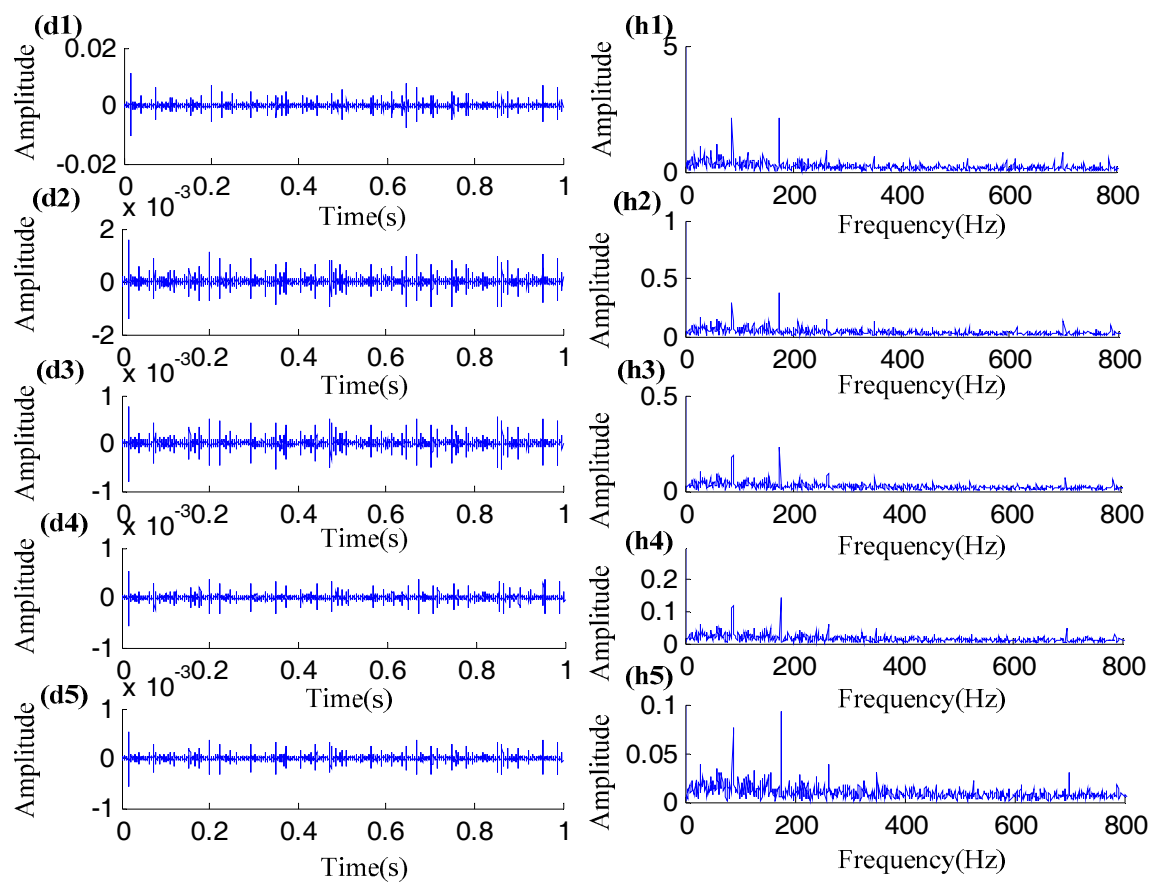

Figure 13. Time domain waveform of the five detail signals with the maximum kurtosis value and their corresponding Hilbert envelope demodulation spectrum. (d1-d5) The five detail signals with maximum kurtosis values obtained using MRSVD decomposition. (h1-h5) The corresponding Hilbert envelope demodulation spectrums of the five detail signals in (d1-d5).

\section{Conclusions}

In this study, a new technique based on MRSVD has been developed for the initial fault detection of a rolling bearing. With this method, the raw bearing vibration signal acquired at the incipient failure stage, which contains the background noise, the harmonic interference, and the periodic impulse characteristics, can be decomposed to a series of approximate signals and detailed signals with different resolutions. Thus, the background noise, the harmonic interference, and the periodic impulse characteristics will correspond to different signal components. Theoretical and experimental analyses show that the first detail signal is mainly composed of noise and the last approximate signal is mainly 
composed of harmonic interference. Therefore, the periodic impulse characteristics that are related to the rolling bearing failure can be extracted from the detail signals (in addition to the first detail signal).

The advantages of the introduced approach include the following: (a) in contrast to the resonant demodulation approaches, this approach extracts the periodic impulse characteristics for demodulation analysis in order to identify the fault without relying on prior knowledge of the fault-excited resonance frequency; (2) this approach is computationally efficient, as except for the singular value decomposition of a two-row matrix, the algorithm only involves addition and subtraction.

Author Contributions: The topic of this research is designed by J.L. and S.Z.; Formal analysis is completed by J.L.; The first version of manuscript is prepared by J.L.; and this version of the manuscript is read, approved and substantially contributed by all authors.

Funding: This work was supported by the Natural Science Foundation of China (grant no. 51605405, 51975121, 51605406), the Science and Technology Innovation Project of Fujian Province, China (grant no. 2016H2003), the Natural Science Foundation of Fujian Province (grant no. 2018J01531), and the program for Innovative Research Team in Science and Technology at the Fujian Province University. Their support is gratefully acknowledged.

Acknowledgments: The authors wish to thank $Y$ Wang and $Y$ Zhang for their experimental work carried out at the University of Ottawa lab. They also wish to thank the Board member and the reviewer for their valuable suggestions and comments that have helped improve this paper.

Conflicts of Interest: The authors declare no conflict of interest.

\section{References}

1. Randall, R.B.; Antoni, J. Rolling element bearing diagnostics-A tutorial. Mech. Syst. Signal Process. 2011, 25, 485-520. [CrossRef]

2. Jaskaran, S.; Ashish, D.; Satinder, P.S. Rolling element bearing fault detection based on Over-Complete rational dilation wavelet transform and auto-correlation of analytic energy operator. Mech. Syst. Signal Process. 2018, 100, 662-693.

3. Yaqub, M.F.; Gondal, I.; Kamruzzaman, J. Inchoate Fault Detection Framework: Adaptive Selection of Wavelet Nodes and Cumulant Orders. IEEE Trans. Instrum. Meas. 2012, 61, 685-695. [CrossRef]

4. Zhang, W.; Peng, G.; Li, C. Rolling element bearings fault intelligent detection based on convolutional neural networks using raw sensing signal. In Advances in Intelligent Information Hiding and Multimedia Signal Processing; Springer International Publishing: Cham, Germany, 2017.

5. Jiang, X.; Li, S.; Cheng, C. A novel method for adaptive multiresonance bands detection based on VMD and using MTEO to enhance rolling element bearing fault detection. Shock Vib. 2016, 2016, 7-20. [CrossRef]

6. Cui, L.; Huang, J.; Zhang, F. Quantitative and localization detection of a defective ball bearing based on Vertical-Horizontal synchronization signal analysis. IEEE Trans. Ind. Electron. 2017, 64, 8695-8705. [CrossRef]

7. Zhang, S.; Sun, Z.; Wang, M.; Long, J.; Bai, Y.; Li, C. Deep Fuzzy Echo State Networks for Machinery Fault Diagnosis. IEEE Trans. Fuzzy Syst. 2019. [CrossRef]

8. Zhang, S.; Sun, Z.; Li, C.; Cabrera, D.; Long, J.; Bai, Y. Deep hybrid state network with feature reinforcement for intelligent fault diagnosis of delta 3D printers. IEEE Trans. Ind. Inform. 2019. [CrossRef]

9. Wang, Y.; He, Z.; Zi, Y. A comparative study on the local mean decomposition and empirical mode decomposition and their applications to rotating machinery health detection. J. Vib. Acoust. 2010, 132, 613-624. [CrossRef]

10. Yan, R.; Gao, R.X.; Chen, X. Wavelets for fault detection of rotary machines: A review with applications. Signal Process. 2014, 96, 1-15. [CrossRef]

11. Wang, H.; Chen, J.; Dong, G. Feature extraction of rolling bearing's early weak fault based on EEMD and tunable Q-factor wavelet transform. Mech. Syst. Signal Process. 2014, 48, 103-119. [CrossRef]

12. Deng, L.; Zhao, R. Fault feature extraction of a rotor system based on local mean decomposition and Teager energy kurtosis. J. Mech. Sci. Technol. 2014, 28, 1161-1169. [CrossRef]

13. Wang, S.; Chen, X.; Tong, C.; Zhao, Z. Matching synchrosqueezing wavelet transform and application to aeroengine vibration monitoring. IEEE Trans. Instrum. Meas. 2017, 66, 360-372. [CrossRef]

14. Li, C.; Liang, M. Time-frequency signal analysis for gearbox fault detection using a generalized synchrosqueezing transform. Mech. Syst. Signal Process. 2012, 26, 205-217. [CrossRef] 
15. Osman, S.; Wang, W. A leakage-free resonance sparse decomposition technique for bearing fault detection in gearboxes. Meas. Sci. Technol. 2018, 29, 035004. [CrossRef]

16. Cui, L.; Gong, X.; Zhang, J.; Wang, H. Double-dictionary matching pursuit for fault extent evaluation of rolling bearing based on the Lempel-Ziv complexity. J. Sound Vib. 2016, 385, 372-388. [CrossRef]

17. Ding, X.; He, Q. Time-frequency manifold sparse reconstruction: A novel method for bearing fault feature extraction. Mech. Syst. Signal Process. 2016, 80, 392-413. [CrossRef]

18. Zhang, S.; Sun, Z.; Long, J.; Li, C.; Bai, Y. Dynamic condition monitoring for 3D printers by using error fusion of multiple sparse auto-encoders. Comput. Ind. 2019, 105, 164-176. [CrossRef]

19. Wang, Y.; Xiang, J.; Markert, R.; Liang, M. Spectral kurtosis for fault detection, diagnosis and prognostics of rotating machines: A review with applications. Mech. Syst. Signal Process. 2016, 66-67, 679-698. [CrossRef]

20. Liang, M.; Bozchalooi, I.S. An energy operator approach to joint application of amplitude and frequencydemodulations for bearing fault detection. Mech. Syst. Signal Process. 2010, 24, 1473-1494. [CrossRef]

21. Rodríguez, P.H.; Alonso, J.B.; Ferrer, M.A. Application of the Teager-Kaiser energy operator in bearing fault detection. ISA Trans. 2013, 52, 278-284. [CrossRef]

22. Wang, H.; Gao, G.G.; Zeng, X.W. Wind Turbine Gearbox Fault detection Based on Wavelet Theory and Hilbert Demodulation Spectrum. Appl. Mech. Mater. 2015, 703, 390-393. [CrossRef]

23. Liu, X.; Bo, L.; Peng, C. Application of order cyclostationary demodulation to damage detection in a direct-driven wind turbine bearing. Meas. Sci. Technol. 2014, 25, 25004-25011. [CrossRef]

24. Wang, C.; Jiang, D.P.; He, Z.C. Research on fault diagnoses of rolling bearing based on the energy operator demodulation approach. Adv. Mater. Res. 2012, 588-589, 134-140. [CrossRef]

25. Zhou, Y.; Chen, J.; Dong, G.M.; Xiao, W.B.; Wang, Z.Y. Application of the horizontal slice of cyclic bispectrum in rolling element bearings detection. Mech. Syst. Signal Process. 2012, 26, 229-243. [CrossRef]

26. Saidi, L.; Ali, J.B.; Fnaiech, F. Application of higher order spectral features and support vector machines for bearing faults classification. ISA Trans. 2015, 54, 193-206. [CrossRef]

27. Yunusa-Kaltungo, A.; Sinha, J.K. A comparison of signal processing tools: Higher order spectra versus higher order coherences. J. Vib. Eng. Technol. 2015, 3, 461-472.

28. Yang, D.M.; Stronach, A.F.; MacConnell, P.; Penman, J. Third-order spectral techniques for the detection of motor bearing condition using artificial neural networks. Mech. Syst. Signal Process. 2002, 16, 391-411. [CrossRef]

29. Yunusa-Kaltungo, A.; Sinha, J.K.; Nembhard, A.D. Use of composite higher order spectra for faults detection of rotating machines with different foundation flexibilities. Measurement 2015, 70, 47-61. [CrossRef]

30. Li, F.; Meng, G.; Ye, L.; Chen, P. Wavelet transform-based higher-order statistics for fault detection in rolling element bearings. J. Vib. Control 2008, 14, 1691-1709.

31. Howard, I.M. Higher-order spectral techniques for machine vibration condition monitoring. Proc. Inst. Mech. Eng. Part G J. Aerosp. Eng. 1997, 211, 211-219. [CrossRef]

32. Shao, R.; Li, J.; Hu, W.; Dong, F. Multi-fault clustering and detection of gear system mined by spectrum entropy clustering based on higher order cumulants. Rev. Sci. Instrum. 2013, 84, 025107. [CrossRef] [PubMed]

33. Yunusa-Kaltungo, A.; Sinha, J.K. Sensitivity analysis of higher order coherent spectra in machine faults detection. Struct. Health Monit. 2016, 15, 555-567. [CrossRef]

34. Sheen, Y.T. On the study of applying Morlet wavelet to the Hilbert transform for the envelope detection of bearing vibrations. Mech. Syst. Signal Process. 2009, 23, 1518-1527. [CrossRef]

35. Han, G.D.; Wan, S.T.; Lv, Z.J.; Liu, R.H.; Wang, J.; Tang, G.J. The Analysis of Gearbox Fault detection Research Based on the EMD and Hilbert Envelope Demodulation. Adv. Mater. Res. 2014, 926-930, 1800-1805. [CrossRef]

36. Luo, J.; Yu, D.; Liang, M. A kurtosis-guided adaptive demodulation technique for bearing fault detection based on tunable-Q wavelet transform. Meas. Sci. Technol. 2013, 24, 055009. [CrossRef]

37. Chibani, A.; Chadli, M.; Ding, S.X.; Braiek, N.B. Design of robust fuzzy fault detection filter for polynomial fuzzy systems with new finite frequency specifications. Automatica 2018, 93, 42-54. [CrossRef]

38. Chadli, M.; Davoodi, M.; Meskin, N. Distributed state estimation, fault detection and isolation filter design for heterogeneous multi-agent linear parameter-varying systems. IET Control Theory Appl. 2016, 11, 254-262. [CrossRef]

39. Zhu, X.; Xia, Y.; Chai, S.; Shi, P. Fault detection for vehicle active suspension systems in finite-frequency domain. IET Control Theory Appl. 2019, 13, 387-394. [CrossRef] 
40. Li, L.; Chadli, M.; Ding, S.X.; Qiu, J.; Yang, Y. Diagnostic observer design for $\mathrm{t}-\mathrm{s}$ fuzzy systems: Application to real-time-weighted fault-detection approach. IEEE Trans. Fuzzy Syst. 2017, 26, 805-816. [CrossRef]

41. Tian, Y.; Ma, J.; Lu, C.; Wang, Z. Rolling bearing fault detection under variable conditions using LMD-SVD and extreme learning machine. Mech. Mach. Theory 2015, 90, 175-186. [CrossRef]

42. Jiang, H.; Chen, J.; Dong, G.; Liu, T.; Chen, G. Study on Hankel matrix-based SVD and its application in rolling element bearing fault detection. Mech. Syst. Signal Process. 2015, 52-53, 338-359. [CrossRef]

43. Shen, F.; Chen, C.; Yan, R.; Gao, R.X. Bearing fault detection based on SVD feature extraction and transfer learning classification. In Proceedings of the Prognostics and System Health Management Conference (PHM), Beijing, China, 21-23 October 2015.

44. Liao, Z.; Song, L.; Chen, P.; Guan, Z.; Fang, Z.; Li, K. An Effective Singular Value Selection and Bearing Fault Signal Filtering detection Method Based on False Nearest Neighbors and Statistical Information Criteria. Sensors 2018, 18, 2235. [CrossRef] [PubMed]

45. Qiao, Z.; Pan, Z. SVD principle analysis and fault detection for bearings based on the correlation coefficient. Meas. Sci. Technol. 2015, 26, 085014. [CrossRef]

46. Zhao, X.; Ye, B.; Chen, T. Theory of multi-resolution singular value decomposition and its application to signal processing and fault detection. J. Mech. Eng. 2010, 46, 64-75. [CrossRef]

(C) 2019 by the authors. Licensee MDPI, Basel, Switzerland. This article is an open access article distributed under the terms and conditions of the Creative Commons Attribution (CC BY) license (http://creativecommons.org/licenses/by/4.0/). 